\title{
First-principles characterization of reversible martensitic transformations
}

\author{
Alberto Ferrari, ${ }^{1, *}$ Davide G. Sangiovanni, ${ }^{1,2}$ Jutta Rogal, ${ }^{1}$ and Ralf Drautz ${ }^{1}$ \\ ${ }^{1}$ Interdisciplinary Centre for Advanced Materials Simulation, Ruhr-Universität Bochum, 44801 Bochum, Germany \\ ${ }^{2}$ Department of Physics, Chemistry, and Biology (IFM), Linköping University, SE-58183 Linköping, Sweden
}

(Received 16 October 2018; published 19 March 2019)

\begin{abstract}
Reversible martensitic transformations (MTs) are the origin of many fascinating phenomena, including the famous shape memory effect. In this work, we present a fully ab initio procedure to characterize MTs in alloys and to assess their reversibility. Specifically, we employ ab initio molecular dynamics data to parametrize a Landau expansion for the free energy of the MT. This analytical expansion makes it possible to determine the stability of the high- and low-temperature phases, to obtain the Ehrenfest order of the MT, and to quantify its free energy barrier and latent heat. We apply our model to the high-temperature shape memory alloy Ti-Ta, for which we observe remarkably small values for the metastability region (the interval of temperatures in which the highand low-temperature phases are metastable) and for the barrier: these small values are necessary conditions for the reversibility of MTs and distinguish shape memory alloys from other materials.
\end{abstract}

DOI: 10.1103/PhysRevB.99.094107

\section{INTRODUCTION}

A martensitic transformation (MT) [1] is a diffusionless phase transition, triggered by temperature or stress, which changes the symmetry of a high-temperature phase (austenite) and forms variants of a low-temperature phase (martensite). Most of the MTs are irreversible, as dislocations, shear, and plastic deformation accumulate during the transformation. However, if the symmetry of martensite is lower than that of austenite and if the variations in lattice parameters and atomic volumes are small, the MT can be reverted, that is, the system can be switched between the two phases with small latent heat [2-5]. Reversible MTs in metals or polymers are appealing as they often result in the shape memory effect, the ability to recover a predetermined shape upon heating, and pseudoelasticity, the capacity to accommodate large deformations without plasticity [6-8]. Other examples in which reversible MTs are important include the recently discovered gum metals [9], where metastable phases have been observed to form via reversible transformations [10].

An urgent technological challenge for actuator and biomedical applications is to identify alloys that exhibit reversible MTs that are stable during operational cycles. With very few exceptions [11], first-principles investigations aiming to clarify the mechanisms underlying a MT generally rely on static, $T=0 \mathrm{~K}$ calculations. These, however, are often inadequate to describe the atomistic processes responsible for the dynamic and/or thermodynamic stabilization of the austenite phase at finite temperatures, as well as the interval of temperatures in which austenite and martensite are metastable (metastability region), the free energy barrier, the latent heat, and even the Ehrenfest order of a MT.

To overcome these limitations we have employed $a b$ initio molecular dynamics (aiMD) simulations to access structural

\footnotetext{
*alberto.ferrari@rub.de
}

properties at finite temperature, and combined our $a b$ initio data with a 2-4-6 Landau-Falk expansion of the free energy $[12,13]$ to characterize the nature of reversible MTs and suggest necessary conditions to distinguish them from irreversible ones. We have applied our method to the shape memory alloy Ti-Ta [14-23] that features a reversible MT with a high $\left(>100^{\circ} \mathrm{C}\right)$ transition temperature. Our key findings include that, in this system, there is only a small interval of temperatures where austenite and martensite are both dynamically stable and that, in this interval, the two phases are separated by an extremely small free energy barrier.

Any first-order phase transition, such as the reversible MT described here, involves the nucleation and growth of a new phase inside the other; the consideration of this mechanism is beyond the scope of this work. Nevertheless, even for a homogeneous transition, small metastability regions, energy barriers, and latent heats generally distinguish reversible MTs from ordinary MTs; with our approach we provide a fully $a b$ initio strategy to identify these fundamental characteristics of a MT.

\section{COMPUTATIONAL DETAILS}

We have performed Parrinello-Rahman NPT molecular dynamics [24,25] with a Langevin thermostat and an Andersen barostat with Langevin friction [26], as implemented in the Vienna Ab initio Simulation Package (VASP 5.4) [27-29]. The friction coefficients of both the Langevin thermostat and the barostat have been set to $\gamma=0.1 \mathrm{ps}^{-1}$, while a value of $M=1$ a.m.u. has been used for the mass of the extended particle in the Andersen barostat. With these settings, the root mean squared deviation of the instantaneous $T$ and $P$ from their average values was of the order of $20 \mathrm{~K}$ and $100 \mathrm{MPa}$, respectively. A time step of $1 \mathrm{fs}$ has been employed for all simulations. Sampling has always been started after complete equilibration of both temperature and pressure, and 
thermodynamic averages have been obtained from trajectories with a duration of at least $7 \mathrm{ps}$.

Total energies and forces have been computed using density functional theory (DFT) with projector-augmented wave (PAW) [30,31] pseudopotentials including $s, p$, and $d$ electrons for $\mathrm{Ti}$ and $\mathrm{Ta}$. The generalized gradient approximation (GGA) functional parametrized by Perdew, Burke, and Ernzerhof (PBE) [32] has been utilized for the exchangecorrelation term. To integrate the Brillouin zone, we have employed the Monkhorst-Pack scheme $[33,34]$ with a $k$-point mesh with a linear density of $0.32 \pi / \AA$. The electronic occupations have been smeared with the Methfessel-Paxton method [35] with a width of $0.05 \mathrm{eV}$. The energy cutoff has been fixed to $400 \mathrm{eV}$. These settings have been found to ensure an accuracy of approximately $4 \mathrm{meV} /$ at. on total energy differences. NPT simulations change the volume of the supercell and therefore imply the presence of Pulay stresses if plane-wave basis sets are used [36]. In our calculations, we estimate that the absolute value of the volume is systematically underestimated by roughly $0.5 \%$ with respect to static calculations of the equilibrium volume with the Birch-Murnaghan equation of state $[37,38]$. The structural relaxations at $0 \mathrm{~K}$ have been performed on both the atomic and lattice degrees of freedom until all the forces were less than $0.01 \mathrm{eV} / \AA$ and all the components of the stress tensor less than $100 \mathrm{MPa}$.

The simulations have been carried out in $(4 \times 4 \times 4)$ orthorhombic supercells containing 256 atoms. Since Ti-Ta is a solid solution (see next section), the occupations of the lattice sites have been determined according to special quasirandom structures (SQS) configurations $[39,40]$ generated with the Monte Carlo algorithm of a modified version [41,42] of the ATAT package [43]. In the minimization algorithm, geometrical correlations of pair, three-body, four-body, and five-body figures have been considered up to the ninth, fifth, fourth, and second neighbor shells, respectively.

\section{MARTENSITIC TRANSFORMATION IN TI-TA}

The austenitic phase in Ti-Ta is a solid solution of Ti and Ta with body-centered cubic (bcc) symmetry, called $\beta$ phase. At lower temperatures the $\beta$ phase breaks its cubic symmetry and transforms into one of the sixfold degenerate orthorhombic martensitic variants, called $\alpha^{\prime \prime}$. As depicted in Fig. $1, \alpha^{\prime \prime}$ (right panel) is obtained from $\beta$ (left panel) by an orthorhombic cell distortion and a displacement of alternating $\{110\}$ atomic planes along $\langle-110\rangle$ directions. The lattice vectors of the martensitic phase are $\left(a_{\alpha^{\prime \prime}}, 0,0\right),\left(0, b_{\alpha^{\prime \prime}}, 0\right),\left(0,0, c_{\alpha^{\prime \prime}}\right)$, with $a_{\alpha^{\prime \prime}}<c_{\alpha^{\prime \prime}} / \sqrt{2}<b_{\alpha^{\prime \prime}} / \sqrt{2}$. The MT in Ti-Ta can be described by two order parameters that change together: the spontaneous lattice strain (SLS) of martensite, which accounts for the respective elongation and shrinkage of the lattice parameters, and the average displacement from ideal bcc positions $\Delta y$. The SLS is given by [22]:

$$
\mathrm{SLS}=2 \cdot \frac{b_{\alpha^{\prime \prime}} / \sqrt{2}-a_{\alpha^{\prime \prime}}}{b_{\alpha^{\prime \prime}} / \sqrt{2}+a_{\alpha^{\prime \prime}}}
$$

and $\Delta y$ is the average relative distance of the atoms in the gliding planes from the ideal bcc positions.

We have performed our calculations for two compositions Ti-25Ta and Ti-31.25Ta, with 25 at.\% and 31.25 at.\% Ta,

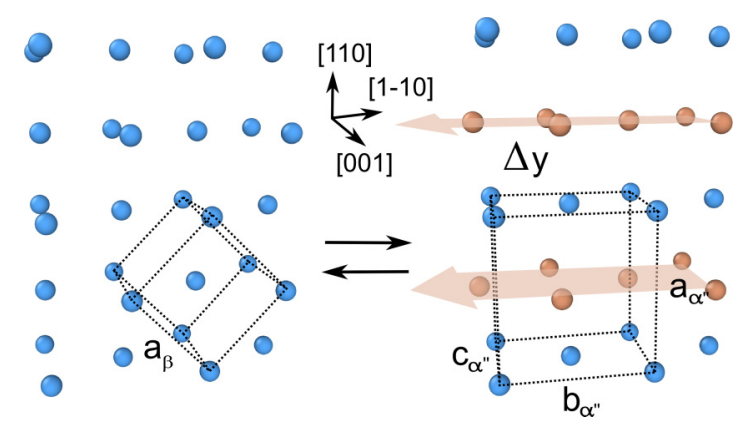

FIG. 1. The MT in Ti-Ta. The austenitic phase (left) is a bcc structure. The martensitic phase (right) is orthorhombic, and it is obtained from the austenitic phase by cell distortion and gliding of alternating $\{110\}$ planes (in brown) along the $\langle-110\rangle$ direction, described by the parameter $\Delta y$.

respectively. For Ti-25Ta we have carried out aiMD simulations at $500 \mathrm{~K}, 600 \mathrm{~K}, 650 \mathrm{~K}$, and $700 \mathrm{~K}$, whereas for Ti-31.25Ta at $230 \mathrm{~K}, 415 \mathrm{~K}, 500 \mathrm{~K}$, and $600 \mathrm{~K}$.

In Fig. 2 the average lattice parameters $a, b / \sqrt{2}$, and $c / \sqrt{2}$ extracted from the aiMD simulations are presented as a function of temperature, and compared to previous $T=0 \mathrm{~K}$ calculations [20] and experimental data on bulk samples [21] and thin films [22]. At low temperature the structures correspond to the orthorhombic $\alpha^{\prime \prime}$ phase, as $a<c / \sqrt{2}<b / \sqrt{2}$ for both compositions. Our $0 \mathrm{~K}$ relaxed lattice constants are generally in very good agreement with the values by Chakraborty et al. [20], and the aiMD simulation results compare very well with the experimental data at room temperature by Kadletz et al. [21,22]. At $T>600 \mathrm{~K}$ and $T \geqslant 500 \mathrm{~K}$ for Ti-25Ta and Ti-31.25Ta, respectively, $b$ and $c$ become equal, indicating that the austenitic phase is formed. The fact that the lattice parameter $a$ is slightly smaller than $b / \sqrt{2}$ and $c / \sqrt{2}$ even at high temperatures, when the system is in the austenitic phase, is due to finite-size effects.

The results for the SLS from the numerical simulations are shown as square symbols in Fig. 3(a). The values of the calculated SLS are consistent with the experimental data. At high temperatures the residual SLS is around $1 \%$, suggesting that the mentioned size effects are small.

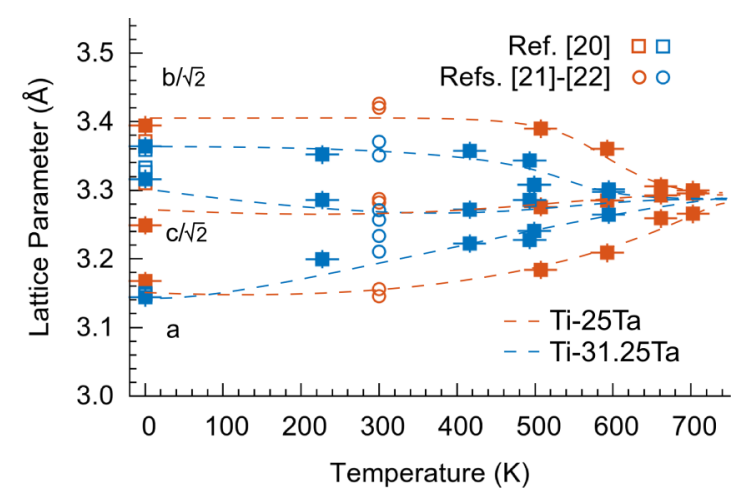

FIG. 2. Lattice parameters of Ti-25Ta (red) and Ti-31.25Ta (blue) as a function of temperature. Circles are experimental data on bulk samples and thin films at room temperature. Empty squares are DFT calculations from Ref. [20]. Broken lines are guides to the eye. 
(a)

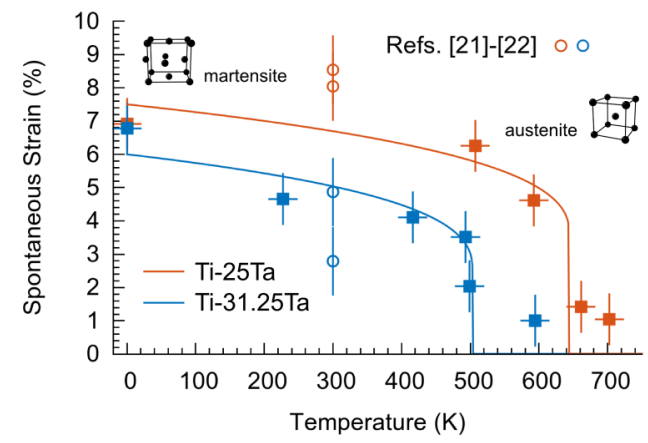

(b)

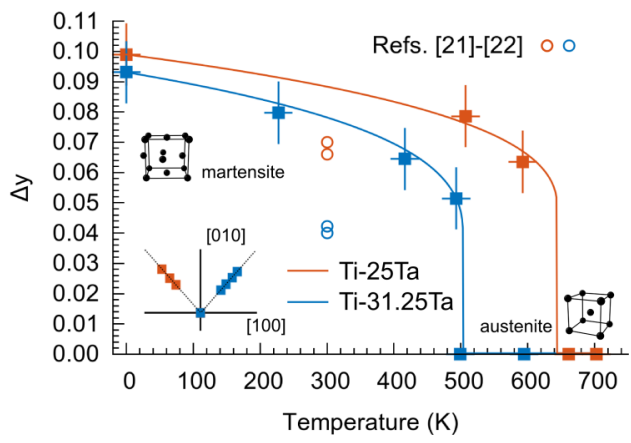

FIG. 3. (a) Spontaneous lattice strain of $\alpha^{\prime \prime}$ for Ti-25Ta (red) and Ti-31.25Ta (blue) as a function of temperature. (b) The time- and atom-averaged $\Delta y$ parameter as a function of temperature for Ti-25Ta (red) and Ti-31.25Ta (blue). The inset shows the directions of the average atomic displacements observed in the aiMD simulations. For both order parameters, squares are extracted from the aiMD simulations, circles are experimental data for bulk and thin film samples at room temperature, and solid lines are predictions from the Landau-Falk expansion (no fit).

The square symbols in Fig. 3(b) represent the atomic displacements $\Delta y$, averaged over time and over all atoms in the supercell, as a function of temperature. For both compositions, at low temperature the value of $\Delta y$ is approximately 0.1 . At $T>600 \mathrm{~K}$ and $T \geqslant 500 \mathrm{~K}$ for Ti-25Ta and Ti-31.25Ta, respectively, $\Delta y$ drops to zero, which indicates that the average atomic positions coincide with those of an ideal bcc lattice. The inset of Fig. 3(b) shows that the displacements for both compositions are in the $\langle 110\rangle$ direction, consistent with the mechanism depicted in Fig. 1. The deviation of the theoretical $\Delta y$ values from the experimental data is attributed to the presence of phase separation in both the bulk and thin film samples in the experiments [44]. Phase separation implies that the Ta content in the $\alpha^{\prime \prime}$ phase is considerably higher than the nominal composition of the samples and leads to a severe underestimation of the $\Delta y$ value.

The transition temperatures have been determined from the simulations by considering the temperature dependence of the two order parameters. For Ti-25Ta both order parameters drop to zero between $600 \mathrm{~K}$ and $650 \mathrm{~K}$, while for Ti-31.25Ta the drop occurs at roughly $500 \mathrm{~K}$. By averaging the actual temperatures of the MD runs, the values for the transition temperatures have been calculated to be $T_{0}=627 \mathrm{~K}$ and $496 \mathrm{~K}$ for the two compositions, respectively.

Compared with the experimental transition temperatures (560 $\mathrm{K}$ and $420 \mathrm{~K}$, respectively [23]), our results slightly overestimate $T_{0}$; an even more severe overestimation has been noted before in aiMD simulations of the shape memory alloy NiTi [11] and imputed to the absence of crystal defects and internal stresses in the calculations. Our values should therefore be considered as an upper limit for $T_{0}$ in an ideal, defect-free crystal. As an additional possible source of error, the finite size of the simulation cell may induce artificial correlations that might result in a shift of the transition temperature and in inaccuracies in the values of the order parameters near $T_{0}$ [45].

\section{A LANDAU EXPANSION OF THE FREE ENERGY}

To fully characterize the MT $\alpha^{\prime \prime} \rightleftharpoons \beta$ we can parametrize the free energy $F(V, T)$, which, at zero pressure, governs the thermodynamics of the phase transition. For reversible MTs, Falk [12] has suggested a 2-4-6 Landau expansion of $F(V, T)$ as a function of a one-dimensional order parameter $\eta$

$$
F(\eta, T)=a \eta^{6}-b \eta^{4}+c\left(T-T_{c}\right) \eta^{2},
$$

where $a, b$, and $c$ are material-dependent parameters, and $T_{c}<T_{0}$ is the temperature at which the austenitic phase becomes metastable. In this picture, $T_{0}$ is the temperature at which the free energies of austenite and martensite are equal. A generalized version of Eq. (2) has been employed in Refs. $[46,47]$ for the cubic-tetragonal phase transition in $\mathrm{ZrO}_{2}$ and in Refs. [48-50] for stress-induced MT.

In the case of the MT in Ti-Ta, Eq. (2) provides a onedimensional description of the relative stability of austenite and one of the sixfold degenerate martensitic variants; $\eta$ can be either the SLS or $\Delta y$, as in the MT the lattice constants and atomic positions are observed to change together.

Traditionally, Eq. (2) has been used to fit order parameters and latent heats measured experimentally. Here, we determine the parameters $a, b, c$, and $T_{c}$ exclusively from first-principles simulation data by imposing the following conditions:

(i) at $0 \mathrm{~K}, F(\eta, T)$ has two minima at respectively $\eta=-1$ and $\eta=+1$;

(ii) at $0 \mathrm{~K}, \quad F(0,0)-F(1,0)=\Delta E^{\left(\beta-\alpha^{\prime \prime}\right)}$, where $\Delta E^{\left(\beta-\alpha^{\prime \prime}\right)}$ is the $0 \mathrm{~K}$ energy difference between austenite and martensite;

(iii) at the transition temperature $T_{0}, F\left(\eta, T_{0}\right)$ has two minima at, respectively, $\eta=-\eta_{0}$ and $\eta=+\eta_{0}$, where $\eta_{0}$ is the value of the order parameter at $T_{0}$ extracted from the aiMD NPT simulations;

(iv) at the transition temperature $T_{0}, \quad F\left(0, T_{0}\right)-$ $F\left(\eta_{0}, T_{0}\right)=0$.

To compute the total energy of austenite at $0 \mathrm{~K}$, we have employed the average positions of the aiMD run at $700 \mathrm{~K}$ for Ti-25Ta and at $600 \mathrm{~K}$ for Ti-31.25Ta. In fact, the chemical disorder in Ti-Ta implies that in the austenitic phase the average atomic positions do not correspond exactly to the perfect bcc positions. 
TABLE I. Columns 2-4: input values for the parametrization of the free energy. Columns 5-8: coefficients of the Landau-Falk expansion. Columns 9-10: conversion factors for the two order parameters.

\begin{tabular}{lccccccccc}
\hline \hline & $\Delta E^{\left(\beta-\alpha^{\prime \prime}\right)}(\mathrm{meV} /$ at. $)$ & $T_{0}(\mathrm{~K})$ & $\eta_{0}$ & $a(\mathrm{meV} / \mathrm{at})$. & $b(\mathrm{meV} /$ at. $)$ & $c(\mu \mathrm{eV} / \mathrm{at} . / \mathrm{K})$ & $T_{c}(\mathrm{~K})$ & $\mathrm{SLS} \mathrm{S}_{0}$ & $\Delta y_{0}$ \\
\hline Ti-25Ta & 40 & 627 & 0.606 & 31.6 & 23.2 & 84.0 & 576 & 7.5 & 0.099 \\
Ti-31.25Ta & 29 & 496 & 0.536 & 20.3 & 11.7 & 79.3 & 475 & 6.0 & 0.093 \\
\hline \hline
\end{tabular}

The input parameters and the values of the coefficients of the free energy expansion are compiled in Table I. An estimation of the accuracy of the extracted parameters is given in the Appendix.

The obtained free energy curves as a function of $\eta$ are presented in Fig. 4 for Ti-25Ta and Ti-31.25Ta at different temperatures. At $0 \mathrm{~K}$ the austenitic phase (corresponding to $\eta=0$ ) is a maximum of the energy, whereas the martensitic phase (corresponding to $\eta= \pm 1$ ) is a minimum. At this temperature there is no barrier separating the two states, meaning that austenite is unstable, in agreement with previous $0 \mathrm{~K}$ static calculations [19]. As the temperature increases, the martensitic minimum shifts towards smaller values of $\eta$. At high temperatures the free energy has only one minimum at the austenitic phase, hence the martensite is unstable. The martensitic and austenitic phases are therefore found to be unstable in a very wide range of temperatures. This is confirmed by our aiMD simulations: as initial configurations we used both the $\alpha^{\prime \prime}$ as well as the $\beta$ phase and apart from the simulations for Ti-31.25Ta at $T=500 \mathrm{~K}$ the structure immediately transformed to the thermodynamically stable one, reflecting the instability of the corresponding other phase.

Within the Landau-Falk expansion, however, a small interval of temperatures around $T_{0}$ is predicted in which both phases are metastable, separated by a very small free energy barrier, as shown in the inset of Fig. 4. Consequently, the phase transition $\alpha^{\prime \prime} \rightleftharpoons \beta$ is of first order, in agreement with experiments [22]. This is also supported by the numerical data: for Ti-31.25Ta at $T \approx 500 \mathrm{~K}$ we have found that the martensitic and austenitic phases coexist. The presence of this free energy barrier is due to entropy contributions to the free energy and cannot be detected with $0 \mathrm{~K}$ calculations.
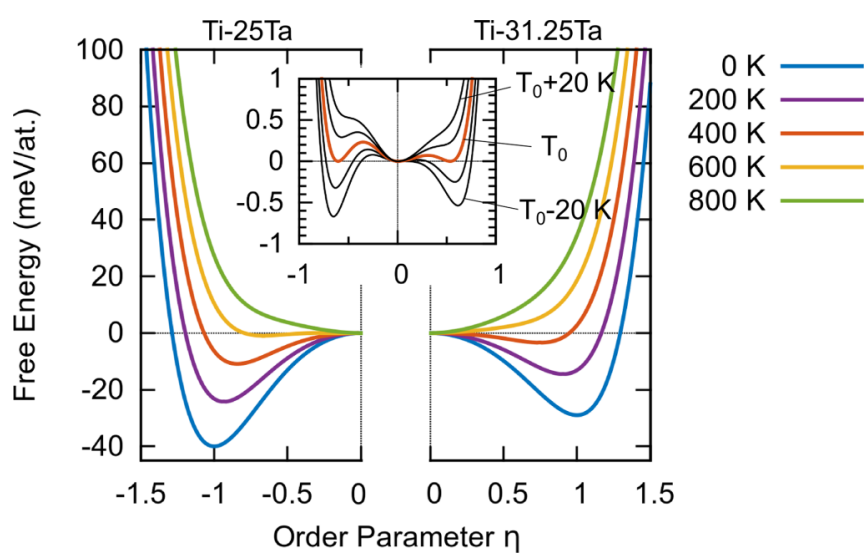

FIG. 4. The free energy profiles as a function of the order parameter for Ti-25Ta (left) and Ti-31.25Ta (right) for different temperatures. The inset shows the same profiles for an interval of temperatures around the transition temperature $T_{0}$.
The entropy difference between austenite and martensite is obtained from Eq. (2) as

$$
S(\eta)=-\frac{\partial F(\eta, T)}{\partial T} .
$$

As can be seen in Fig. 5, where $S$ is plotted as a function of $\eta$, the entropy of the austenitic phase is higher than that of the martensitic phase. This favors the austenitic phase over the martensitic phase at high temperatures. Furthermore, the actual value of the entropy depends very weakly on the composition. This peculiar characteristic of Ti-Ta-based alloys was already assumed in previous work [19,23], where the compositional dependence of the phase stability, which in general depends on both energy and entropy, has been correlated only to $0 \mathrm{~K}$ energy differences, supposing that the entropy difference is constant as a function of the chemical composition.

The entropy difference between austenite and martensite induces a finite latent heat $T_{0} S$ of the MT. We obtain from the Landau-Falk model values of $T_{0} S=19 \pm 3 \mathrm{meV} /$ at. and $11 \pm 3 \mathrm{meV} /$ at. for Ti-25Ta and Ti-31.25Ta, respectively.

The analytical expansion in Eq. (2) can further be used to extract the temperature dependence of the order parameters SLS and $\Delta y$ by imposing

$$
\frac{\partial F(\eta, T)}{\partial \eta}=0
$$

Since $\eta$ is normalized to 1 , the multiplicative factors listed in the last two columns of Table I have been used for the comparison of the analytical predictions of the LandauFalk expansion to the simulation data for SLS and $\Delta y$. The corresponding trends in SLS and $\Delta y$ predicted by the Landau-Falk expansion are presented in Fig. 3 as solid lines. The agreement between the aiMD data and the analytical

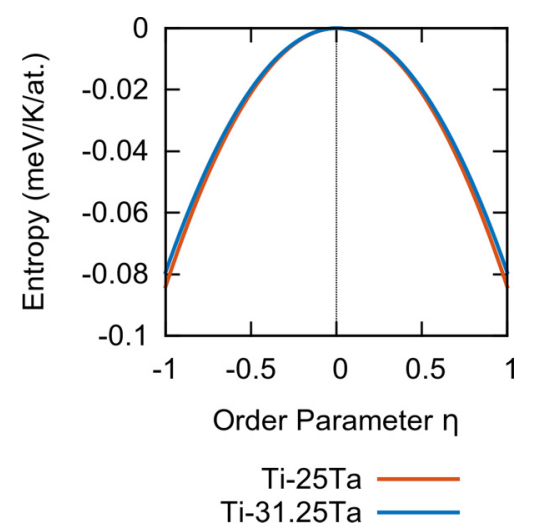

FIG. 5. Entropy as a function of the order parameter for Ti25Ta (red) and Ti-31.25Ta (blue) obtained from the Landau-Falk expansion. 
predictions is remarkable. We would like to stress that the parameters entering Eq. (2) have not been obtained by fitting the temperature dependence of the order parameters SLS and $\Delta y$, but have been extracted from our first-principles data at $0 \mathrm{~K}$ and $T_{0}$. Furthermore, within the Landau-Falk expansion the two order parameters are predicted to be discontinuous at the transition temperature, confirming the first-order character of the MT.

\section{REVERSIBILITY OF THE MARTENSITIC TRANSFORMATION}

The analytical model provides the range of temperatures in which martensite and austenite are both stable (metastability region of the MT)

$$
\Delta T=\frac{b^{2}}{3 a c}
$$

and the height of the barrier at the transition temperature $T_{0}$

$$
\Delta E_{\mathrm{barr}}=-\frac{k \cdot\left[6 a c \cdot\left(T_{0}-T_{c}\right)+b \cdot k\right]}{27 a^{2}},
$$

where

$$
k=-b+\sqrt{b^{2}+3 a c \cdot\left(T_{c}-T_{0}\right)} .
$$

Most notably, we extract from the analytical expansion metastability regions of only $70 \pm 30 \mathrm{~K}$ and $30 \pm 10 \mathrm{~K}$, and free energy barriers of only $200 \pm 70 \mu \mathrm{eV} /$ at. and $100 \pm$ $30 \mu \mathrm{eV} /$ at. for $\mathrm{Ti}-25 \mathrm{Ta}$ and Ti-31.25Ta, respectively. These exceptionally small values indicate that the MT in Ti-Ta is highly reversible. In fact, such small metastability regions and energy barriers for bulk materials are necessary properties that distinguish reversible MTs from irreversible MTs.

A very small free energy barrier is also consistent with our numerical calculations, as for Ti-31.25Ta we have captured a MT within one aiMD run (see Fig. 3 in the Supplemental Material [51]).

According to James' theory $[4,5]$, the reversibility of the MT is quantified by the geometric compatibility of austenite and martensite. If $\mathbf{U}$ is the transformation matrix that connects the martensitic and austenitic lattices and $\lambda_{1}<\lambda_{2}<\lambda_{3}$ are its eigenvalues, then a MT will be more reversible the closer $\operatorname{det} \mathbf{U}$ and $\lambda_{2}$ are to unity. In our case, we find $\operatorname{det} \mathbf{U}=1.001$ and $\lambda_{2}=1.002$ for Ti-25Ta, and $\operatorname{det} \mathbf{U}=1.00002$ and $\lambda_{2}=$ 1.007 for Ti-31.25Ta; therefore, for the two compositions, both conditions for high reversibility are fulfilled. This means that the metastability regions and free energy barriers are so small because the lattices of austenite and martensite are highly compatible with each other. The condition $\operatorname{det} \mathbf{U} \approx 1$ descends from the small difference in atomic volume between martensite and austenite (details are given in the Supplemental Material [51]), and the condition $\lambda_{2} \approx 1$ derives from the small change of the lattice parameter $c / \sqrt{2}$ at the MT, as seen in Fig. 2.

\section{CONCLUSIONS}

In summary, we have successfully applied a combination of ab initio molecular dynamics simulations with an analytical expansion of the free energy to characterize the most significant properties of martensitic transformations, which often cannot be captured by $0 \mathrm{~K}$ calculations. The methodology presented in this work is based entirely on first-principles data and is very well suited to study MTs in a variety of compounds. In particular, we have applied this formalism to the technologically relevant Ti-Ta alloy, for which we have predicted for bulk transformations very small metastability regions (tens of $\mathrm{K}$ ) and very small free energy barriers (hundreds of $\mu \mathrm{eV} / \mathrm{at}$.). These two quantities are decisive in specifying reversible MTs and have to be considered as the fundamental origin of the shape memory effect.

\section{ACKNOWLEDGMENTS}

The work presented in this paper has been financially supported by the Deutsche Forschungsgemeinschaft (DFG) within the research unit FOR 1766 (High Temperature Shape Memory Alloys, http://www.for1766.de, Project No. 200999873, subproject 3). D.G.S. acknowledges financial support from the Olle Engkvist Foundation. The computations have been performed using the Gamma and Triolith clusters, managed by the Swedish National Infrastructure for Computing (SNIC) at the National Supercomputer Centre (NSC) in Linköping, the Kebnekaise cluster at the High Performance Computing Center North (HPC2N) in Umeå, and the Beskow cluster at the Center for High Performance Computing (PDC) in Stockholm.

\section{APPENDIX: ERROR ESTIMATION}

The error bars associated with the values of $T_{0} S, \Delta T$, and $\Delta E_{\text {barr }}$ have been determined by a sensitivity analysis. The factor that influences the latent heat $T_{0} S$ the most is $\Delta E^{\left(\beta-\alpha^{\prime \prime}\right)}$. Deviations of $5 \mathrm{meV} /$ at. on this quantity change the latent heat by roughly $2-3 \mathrm{meV} /$ at., hence a value of $3 \mathrm{meV} /$ at. has been taken as the absolute error in this case. The reported values for $\Delta T$ and $\Delta E_{\mathrm{barr}}$, on the other hand, have been found to be almost insensitive to variations of $5 \mathrm{meV} /$ at. and $50 \mathrm{~K}$ in the parameters $\Delta E^{\left(\beta-\alpha^{\prime \prime}\right)}$ and $T_{0}$. Changes of the order of $5 \%$ on $\eta_{0}$ though affected the final value of these quantities by roughly $30 \%$. This has therefore been assumed as the relative error on $\Delta T$ and $\Delta E_{\text {barr }}$.
[1] M. F. Ashby and D. R. H. Jones, Engineering Materials 2 (Elsevier, Oxford, 1998).

[2] K. Bhattacharya, S. Conti, G. Zanzotto, and J. Zimmer, Nature (London) 428, 55 (2004).

[3] K. Bhattacharya and R. D. James, Science 307, 53 (2005).
[4] R. D. James and Z. Zhang, Magnetism and Structure in Functional Materials, Vol. 79 of Springer Series in Material Science (Springer, New York, 2005).

[5] J. Cui, Y. S. Chu, O. O. Famodu, Y. Furuya, J. Hattrick-Simpers, R. D. James, A. Ludwig, S. Thienhaus, M. Wuttig, Z. Zhang et al., Nature Mater. 5, 286 (2006). 
[6] L. C. Chang and T. A. Read, JOM 3, 47 (1951).

[7] A. Lendlein, A. M. Schmidt, and R. Langer, Proc. Natl. Acad. Sci. USA 98, 842 (2001).

[8] J. M. Jani, M. Leary, A. Subic, and M. A. Gibson, Mater. Des. 56, 1078 (2014).

[9] S. Hao, L. Cui, D. Jiang, X. Han, Y. Ren, J. Jiang, Y. Liu, Z. Liu, S. Mao, Y. Wang et al., Science 339, 1191 (2013).

[10] J. Zhang, C. C. Tasan, M. J. Lai, A. C. Dippel, and D. Raabe, Nature Commun. 8, 14210 (2017).

[11] J. B. Haskins, A. E. Thompson, and J. W. Lawson, Phys. Rev. B 94, 214110 (2016).

[12] F. Falk, Acta Metall. 28, 1773 (1980).

[13] J. Khalil-Allafi, W. W. Schmahl, and T. Reinecke, Smart Mater. Struct. 14, 192 (2005).

[14] Y. A. Bagarjatskii, G. I. Nosova, and T. V. Tagunova, Dokl. Akad. Nauk SSSR 122, 593 (1958).

[15] K. A. Bywater and J. W. Christian, Phylos. Mag. 25, 1249 (1972).

[16] S. G. Fedotov, T. V. Chelidze, Y. K. Kovneristyj, and V. V. Sanadze, Fiz. Met. Metalloved. 60, 567 (1985).

[17] P. J. S. Buenconsejo, H. Y. Kim, H. Hosoda, and S. Miyazaki, Acta Mater. 57, 1068 (2009).

[18] T. Niendorf, P. Krooß, E. Batyrsina, A. Paulsen, Y. Motemani, A. Ludwig, P. Buenconsejo, J. Frenzel, G. Eggeler, and H. J. Maier, Mater. Sci. Eng. A 620, 359 (2015).

[19] T. Chakraborty, J. Rogal, and R. Drautz, J. Phys.: Condens. Matter 27, 115401 (2015).

[20] T. Chakraborty, J. Rogal, and R. Drautz, Phys. Rev. B 94, 224104 (2016).

[21] P. M. Kadletz, Neutron and x-ray diffraction of Ti-Ta and $\mathrm{Co}_{49} \mathrm{Ni}_{21} \mathrm{Ga}_{30}$ high-temperature shape-memory-alloys, Ph.D. thesis, Ludwig-Maximilians-Universität, 2018.

[22] P. M. Kadletz, Y. Motemani, J. Iannotta, S. Salomon, C. Khare, L. Grossmann, H. J. Maier, A. Ludwig, and W. W. Schmahl, ACS Comb. Sci. 20, 137 (2018).

[23] A. Ferrari, A. Paulsen, J. Frenzel, J. Rogal, G. Eggeler, and R. Drautz, Phys. Rev. Mater. 2, 073609 (2018).

[24] M. Parrinello and A. Rahman, Phys. Rev. Lett. 45, 1196 (1980).

[25] M. Parrinello and A. Rahman, J. Appl. Phys. 52, 7182 (1981).

[26] M. P. Allen and D. J. Tildesley, Computer Simulation of Liquids (Oxford University Press, Oxford, 1991).

[27] G. Kresse and J. Hafner, Phys. Rev. B 47, 558 (1993).
[28] G. Kresse and J. Furthmüller, Comput. Mat. Sci. 6, 15 (1996).

[29] G. Kresse and J. Furthmüller, Phys. Rev. B 54, 11169 (1996).

[30] P. E. Blöchl, Phys. Rev. B 50, 17953 (1994).

[31] G. Kresse and D. Joubert, Phys. Rev. B 59, 1758 (1999).

[32] J. P. Perdew, K. Burke, and M. Ernzerhof, Phys. Rev. Lett. 77, 3865 (1996).

[33] A. Baldereschi, Phys. Rev. B 7, 5212 (1973).

[34] H. J. Monkhorst and J. D. Pack, Phys. Rev. B 13, 5188 (1976).

[35] M. P. A. T. Methfessel and A. T. Paxton, Phys. Rev. B 40, 3616 (1989).

[36] P. G. Dacosta, O. H. Nielsen, and K. Kunc, J. Phys. C: 19, 3163 (1986).

[37] F. D. Murnaghan, Proc. Natl. Acad. Sci. USA. 30, 244 (1944).

[38] F. Birch, Phys. Rev. 71, 809 (1947).

[39] A. Zunger, S. H. Wei, L. G. Ferreira, and J. E. Bernard, Phys. Rev. Lett. 65, 353 (1990).

[40] S. H. Wei, L. G. Ferreira, J. E. Bernard, and A. Zunger, Phys. Rev. B 42, 9622 (1990).

[41] J. von Pezold, A. Dick, M. Friák, and J. Neugebauer, Phys. Rev. B 81, 094203 (2010).

[42] J. Koßmann, T. Hammerschmidt, S. Maisel, S. Müller, and R. Drautz, Intermetallics 64, 44 (2015).

[43] A. van de Walle, M. Asta, and G. Ceder, Calphad 26, 539 (2002).

[44] A. Ferrari, P. M. Kadletz, T. Chakraborty, K. Liao, D. Langenkämper, Y. Motemani, A. Paulsen, Y. Lysogorskiy, J. Frenzel, J. Rogal et al., Shap. Mem. Superelasticity 5, 6 (2019).

[45] M. S. S. Challa, D. P. Landau, and K. Binder, Phys. Rev. B 34, 1841 (1986).

[46] S. Fabris, A. T. Paxton, and M. W. Finnis, Phys. Rev. B 61, 6617 (2000).

[47] S. Fabris, A. T. Paxton, and M. W. Finnis, Phys. Rev. B 63, 094101 (2001).

[48] V. I. Levitas and D. L. Preston, Phys. Rev. B 66, 134206 (2002).

[49] V. I. Levitas and D. L. Preston, Phys. Rev. B 66, 134207 (2002).

[50] V. I. Levitas, D. L. Preston, and D.-W. Lee, Phys. Rev. B 68, 134201 (2003).

[51] See Supplemental Material at http://link.aps.org/supplemental/ 10.1103/PhysRevB.99.094107 for more tests on the LandauFalk expansion, which includes also Ref. [52].

[52] D. Sheppard, P. Xiao, W. Chemelewski, D. D. Johnson, and G. Henkelman, J. Chem. Phys. 136, 074103 (2012). 